\title{
Simulation of Working Procedures in a Distribution Centre
}

\author{
M. Santos \\ Dept. Arquitectura de Computadores y Automática \\ CC. Físicas. Universidad Complutense de Madrid. \\ 28040-Madrid. Spain \\ Tel.: +34 91 3944374; Fax: +34913944687 \\ msantos@dacya.ucm.es
}

\author{
J.M. de la Cruz \\ Dept. Arquitectura de Computadores y Automática \\ CC. Físicas. Universidad Complutense de Madrid.
}

\begin{abstract}
This simulation study has been developed to assess a set of realistic proposals of a new distribution centre to a pharmaceutical company, with a focus on the sorting operation. Different sorting strategies, establishing dynamic assignment of the lanes to destinations and lanes to operators are proposed. Also different packing procedures have been presented according to the operator way of working. New qualitative criterions have been defined to evaluate the strategies. The results show some interesting non-linear behaviour in the sorting operation, and give the number of required operators, length of the lanes, and working procedures to be used in this application. The simulation experiments show that the improvement in the overall productivity by choosing a specific sorting strategy can be significant. The study has belped the management of the involved company to make a decision about the supplier and actually, the suggested proposals are being implemented in practice.
\end{abstract}

\section{INTRODUCTION}

Today's distribution centres are facing problems that have been growing in size and complexity over the last several years. As a result, there is an immediate need for procedures in solving various problems encountered in today's distribution operation without extended shutdowns or expensive modifications. Computer simulation is a powerful tool that allows us experimentation with various sorting procedures and different layouts without actual implementation [1].

With the use of simulation, engineers are able to quickly and accurately model future proposed modifications without making costly guesses. Furthermore, if modelled correctly, the simulation model built to assess the significance of a proposed layout change can be evolved into an operational planning tool [2]

This paper presents a practical application of the simulation as a tool to asses a set of realistic proposals of a new distribution centre to a pharmaceutical company. Although there are other simulation approaches in the manufacturing systems [3, 4], simulation is not an usual technique in the pharmaceutical field. First of all, a simulation model of the current distribution system was developed to ascertain its limitations and problems, and to gain the confidence of the management. We focused on the sorting operation. The relevant performance measures from the simulation outputs were analysed to allow us for the identification of a set of operational modifications to the sorting system. Actually, we proposed different sorting strategies based on the combination of:

- different assignment of the lanes to destinations

- different assignment of the lanes to operators
- different operators way of working (packing procedures)

In all of them, we tried to establish completely dynamic assignment, showing the advantages of this method. In order to evaluate these strategies, some new qualitative criterions have been defined.

The simulation experiments showed that the overall productivity can be increased by choosing a specific sorting strategy. The results present also an interesting non-linear behaviour regarding the number of required operators.

The outcome of the simulation study was taken into account by the decision-makers of the involved company to choose the supplier, and the recommended design of the new distribution centre is being implemented in practice. The pharmaceutical distribution enterprise has followed the guidelines that have been come out as a result of this analysis. It is needed to remark that the company had not used these simulation techniques previously.

The paper is organised as follows. Section 2 presents an overview of the performing of the system. Section 3 analyses different sorting strategies and working procedures. In Section 4, the conditions for the simulation runs are explained and the results of different simulation scenarios are shown and discussed. In Section 5 the conclusions are summarised.

\section{OVERVIEW OF THE SYSTEM}

The analysis of a logistic distribution centre has been carried out in the context of a research project for an important pharmaceutical co-operative society, COFARES, leader in the distribution of medicinal, healthcare products and services to the chemist shops in Spain. The main node of the company, the logistic distribution centre, can either supply products directly not only to the chemist's but also to the warehouses, or to both. The geographical setting involves small towns, big cities and huge regions. The role of this main node is to collect the orders of the clients and to respond to them as soon as possible. This centre has to arrange the reception, storage and sorting of the products in order to distribute them to the warehouses that are spread throughout the country. Thus, it performs as a filter, allowing the warehouses to avoid the disturbances and irregularities caused by the suppliers. This centralized strategy makes easier the global forecast of the necessities so it cuts costs and allows the distribution to be optimised.

The distribution centre has a receiving area where the arriving pallets are brought from the trucks. The operators in charge of the unloading check them visually for possible faults in the product. After an intermediate temporal storage, the pallets are sent to an inspection area where, in 
some repacking stations, they are defragmented into the original items (PSL: Packet Standard Laboratory). The operator loads and unloads the product on the test fixture, and does some manual operations over the product guided by the computer via the monitor screen. All the items of the pallet are checked in terms of requested characteristics (price, number of units in each PSL, etc.).

The computer system decides the destination of each PSL according to the customer orders and, for the sorting of the items, a barcode label is printed and applied. The operator puts all the identified items on to the conveying system directed to the sorter.

The items are transported to the entrance of the sorter to be sorted. The items are diverted to their respective outlets, based on the information contained in the barcodes. The sorter consists of several spurs and a main conveyor. Each lane has been assigned an outlet location. When all the items with the same destination are pushed into the spur, the operator sorts the items which are stored, takes an empty container, takes the items and puts them on one pallet. Then he prints a shipping label and applies it on the final pallet which he then puts back in the conveying system. This final pallet is transported to the shipping area where it will be loaded in trucks to reach the different locations. The structure of the distribution centre is shown in Fig. 1, and a general 3D simulation model view can be seen in Fig. 2.

As soon as a truck is available, the pallets coming from one or from different shipping lanes are loaded on it.

The manufacturing system is complex because of the interdependent relationships created by the different operations [5]. The logistic centre performs quite different activities although easily identifiable, which suggests that each one of them can be modelled independently taking into account the coupling between them.

The fact that the model can be utilized for optimising performance parameters and is capable of predicting system performance makes simulation a perfectly suitable tool to tackle the problem under study [6]. For this reason, simulation models for different proposals have been developed, tested, verified and evaluated.

In order to approach these simulations successfully and in time, the necessity of a discrete event simulation tool is evident [7], and the object oriented approach is the most widely applied to manufacturing systerns [8].

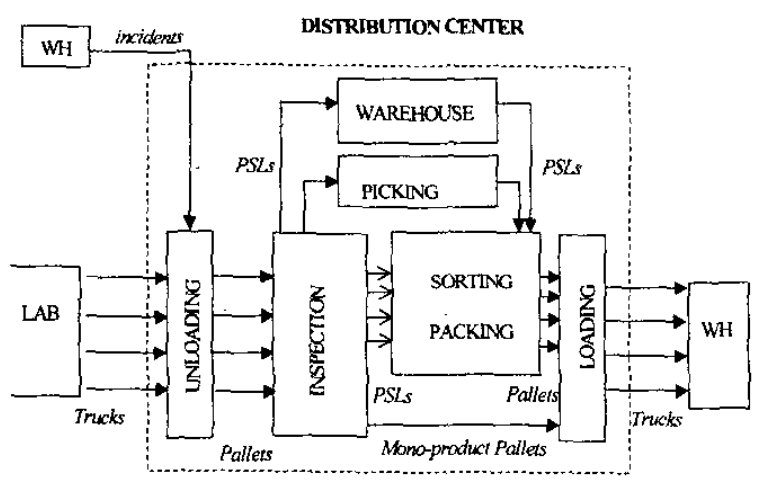

Fig. 1 Modular scheme of the distribution centre and its activities

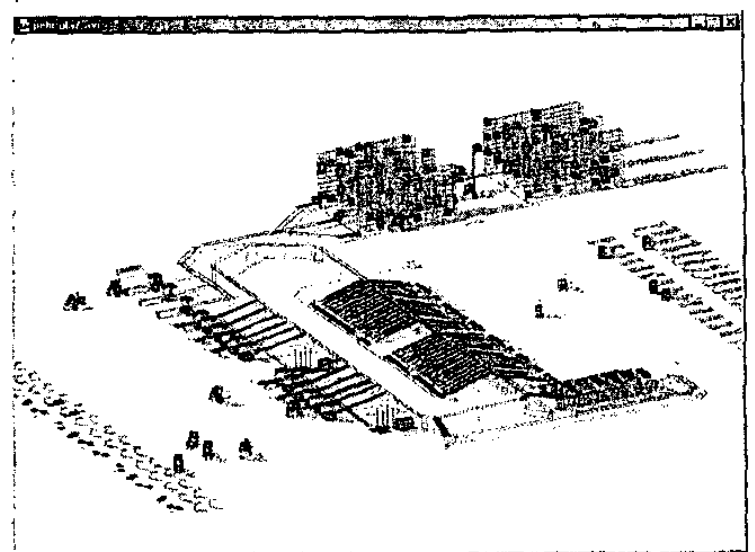

Fig. 2 3D Simulation genesal view of the distribution centre

The elementary requirements are covered by the software package Taylor Ed, Taylor Enterprise Dynamics, by F\& H Simulations B. V. [9], which was selected to carry out the simulation project with COFARES because of its flexibility.

The basic element is called atom [10]. It is an approach to pseudo-object oriented programming. Each atom has a number of events in common (OnEnter, OnExit, OnReset, OnUser, etc.) to reply to. When defining the atom performing, a functional language named 4Dscript is used. It allows us to specify the atom response when an event takes place. The most attractive feature of this programming language is its power; in fact, the program Taylor Ed itself can be considered as a 4Dscript application that is executed by the language interpretation engine. This characteristic, with the fact that the language is interpreted and therefore, their programs are distributed in text files -and so they do not need to be compiled-, makes it a completely configurable application. The graphic appearance (menus, buttons, etc) can be changed by modifying the program code. The application and atoms code is readable, so it facilitates the training by examples.

Another interesting feature, quite important nowadays although it is not essential for simulation, is the 3D capacity. Besides the inherent appeal of $3 \mathrm{D}$ visualization, it can be a very useful tool for the verification and validation of the simulation model, and it also provides an interactive and virtual environment in which credible solutions can be obtained. Together with the statistical analysis of the output values, the visual animation is useful to get a more real approximation of how the system works.

\section{ANALYSIS OF DIFFERENT SORTING AND WORKING STRATEGIES}

The main aim of the project was to advise the involved company on the design of a new distribution centre, focusing on the sorter. The three principal aspects under consideration are: the needed workforce, the structural requirements, and the potential strategies of sorting and packing [11].

The purpose is to establish a completely dynamic assignment for the different locations. That implies to 
decide both: the different operating strategies of the sorter, which can be considered as the heart of the logistic distribution centre, and the operators' way of working, which are in charge of the packaging. Completely dynamic assignment means that there is no previous fixed outlet location for each lane. The item is diverted to the most adequate outlet attending some optimisation aspects. On the other hand, the performing of the workforce has a great influence in choosing the strategy of the sorter.

\section{A. Sorting strategies}

The different proposed sorting strategies can be classified as follows,
A) Destination-Lane Assignment
A.1 Static
A.2 Dynamic
A.2.1 Completely dynamic
A.2.2 Partially dynamic
B) Operator-Lane Assignment
B.1 Completely dynamic
B.2 Partially dynamic

These two points, A) and B), with the packing procedures, are completely independent and have been combined in all the different possible ways for the analysis.

A) Destination-Lane Assignment: initially all the lanes are supposed to be free and therefore available to be assigned to any outlet location. Nevertheless, in some cases may be necessary to limit the lanes that can be assigned (some of them maybe out of order for some reason: repairing, blocked, etc.). Once an outlet has been assigned to a particular destination, all the items with that destination will be diverted to it. The basic assumption is that the same lane cannot be shared by different destinations. So, each spur is assigned to a different location. When the operator finishes packing a pallet up, the lane is empty again and therefore available (free). Otherwise it is busy.

A.1 Static Assignment: the lanes are assigned a fixed destination. This distribution can be changed only if the sorter is reconfigured. The sorter is supposed to have at least equal or more spurs than destinations; otherwise, several locations will share a lane producing a relevant increment of possible conflicts and circulation.

A.2 Dynamic Assignment

A.2.1 Partially dynamic assignment: the lanes are grouped by zones. A particular destination can only be assigned to any of the lanes that belong to a specific group of spurs. If none of them is free in that group, the items will circulate on the sorter until any outlet of that area was free, although there were many other lanes that are free but belonging to other groups of locations.

This option seems adequate for certain situations, for instance, if no destination tends to capture most of the lanes of the sorter. A well defined distribution, taking into account all the necessities of each location, can perform satisfactory although it demands updated daily information. The problem can arise when a seasonal demand takes place in a specific location and the sorter must be reconfigured to supply it. The grouping can be done attending different purposes:

- By geographical reason: the same truck can transport the products to different places, so the outlet destinations for those different locations should be close to facilitate the loading. This criterion is not useful if the pallets are automatically transported to the dispatch area.

- By attending other restrictions such as trying to limit the number of lanes that can be assigned to a particular location to prevent some locations from taking most of the spurs because of their high flow.

- By stabilising criterions: the lanes are grouped so that each group of them has approximately the same load. This solution adds an unnecessary physical constraint.

A.2.2 Completely dynamic assignment: any location can be assigned to any lane without any prior restriction. If there are several free outlets, the item will be diverted to the spur which is nearest to the enter of the sorter, so that to reduce its time in transit, or to the first lane of the corresponding operator so that to balance the operator load.

B) Operator-Lane Assignment: the group of lanes an operator will be in charged of. These groups can be shared by different operators (overlapping) or be exclusive.

B.1 Completely dynamic assignment: any operator can pack at any lane. The one who will do it when required is the one who has been most of his time free until that moment or, if all of them are busy, the first to be released.

B.2 Partially dynamic assignment: each operator is in charge of a group of adjoining lanes to avoid unnecessary movements. He will pack only at his group of lanes.

\section{B. Packing procedures}

They define the operator way of working: 'when' and 'which' lane must be attended to by the operator, and the way of packing. Three different packing procedures have been considered:

1) Complete packing: the operator attends to a lane to get it empty. That can happen because the outlet is full of items (related to the length of the lane) or because it contains all the items missed to complete a pallet (related to the dimension of the pallet). Once he started to pack the items into the container, he continues until he empties the spur out.

2) Half packing: the operator attends to a lane when the items which it contains exceed a threshold that has been previously fixed. He will pick the items on the pallet even if the spur is not full or there were not enough items to complete a pallet.

3) One-to-one packing (continuous): the operator starts packing as soon as an item arrives to the lane. He will remain in that lane until there is no any PSL left. Then he will attend to any other lane that had any item. 
An independent consideration of these points suggests that, in general, the two first options, 1) and 2), will produce the items circulate on the sorter because the length of the lanes is not enough for containing all the items of a pallet. On the other hand, fewer operators are required and they do not need to spend so much time in transit.

The last altemative, 3 , is the one which could be considered more efficient in the sense of avoiding circulation, although it demands more operators and they will spend more time in transit. Therefore their performance will be poorer.

\section{Qualitative evaluation of the procedures}

Although some analytical measurements will be used as evaluation indexes, a qualitative characterization of the different possibilities can be done based on the following four aspects,

- Available Zone (AZ): this parameter is high if, when being a lane free (i.e., it is not assigned to any outlet location) in any place of the sorter, and the coming PSL is diverted to a destination that does not have any spur assigned, the system immediately assigns a free outlet to that item. AZ is low when, although there were free lanes, the item is not diverted to any of them due to some restriction as, for example: previous fixed configuration -only a number of lanes for each location-, some lanes for each destination but not any, etc.

- Loading Balance (LB): the number and distribution of the pallets that are packed during the day at each spur should be approximately the same, i.e., the workload is evenly distributed between the lanes (pallets/lane/day).

- Operator in Transit (OT): this parameter is high if the workforce has to walk very often from one lane to another, even if the distance between them is small.

- Working Balance (WB): it is high if the pallets packed by each operator during the entire shift are the same than the others did, that is, the work load is evenly distributed between the operators (pallets/operator/day).

The parameters $\mathrm{AZ}, \mathrm{WB}$, and $\mathrm{LB}$ mean positive characteristics whereas the time in transit OT is negative in the sense that if it is high, the performance is poorer. These criterions establish a characterisation of the different possibilities that can be considered. These points were a great help in order to make a decision about the final strategy.

Combining the possible ways of performing presented in this section III, the characterisation of the procedures can be summarised as Table I, where the symbol '-' means that the corresponding parameter has a low value, and the symbol 't' refers to high values of that characteristic.

The parameters WB and $L B$ in the first two rows on the left hand could be improved by establishing a fixed distribution to balance the operator load and the lanes. But that requires updated and complete information of the orders everyday.

In general, regarding this qualitative approach, the best option is the algorithm that has been called "Completely dynamic Destination-Lane and partially dynamic OperatorLane assignment".
Table I: Qualitative characterization of the possible assignment strategies

\begin{tabular}{|l|c|c|}
\hline & \multicolumn{2}{|c|}{ Operator-Lane } \\
\hline $\begin{array}{l}\text { Destination } \\
\text { Lane }\end{array}$ & Partially dynamic & Completely dynamic \\
\hline Static & AZ- OT-WB-LB- & AZ-OT+ WB+LB- \\
\hline $\begin{array}{l}\text { Partially } \\
\text { dynamic }\end{array}$ & AZ- OT- WB- LB- & AZ-OT+ WB+LB- \\
\hline $\begin{array}{l}\text { Completely } \\
\text { dynamic }\end{array}$ & AZ+ OT-WB+LB+ & AZ+ OT+ WB+LB+ \\
\hline
\end{tabular}

\section{SIMULATION RUNS}

In the first phase of the simulation study a simulation model of the actual system was developed. In spite of being much simpler, this model was used to: (i) allow for a better understanding of the actual system, (ii) ascertain the critical resources of the system, (iii) gain the confidence of the decision makers regarding the used methodology, and (iv) validate the assumptions made to build the simulation model.

The most interesting combinations of the previously presented alternatives have been implemented for the new distribution centre. Simulations have been carried out, running each one with the three possible ways of working of the operators: complete packing, half packing, one-to-one packing. The results help to confirm the qualitative analysis stated in Table $\mathrm{I}$, that is, the Completely dynamic Destination-Lane, with Partially dynamic Operator-Lane Assignment strategy has been proved, in general, as the most appropriate one, and the one-to-one packing mode as the best workforce way of working.

In consequence, starting from that operation mode, a detailed study has been carried out to analyse the influence of the number and length of the lanes (structural characteristics), and to estimate the necessary number of operators (workforce requirements).

In developing the simulation model particular care was taken to model the distribution process as close to reality as possible. This was accomplished because:

- historical data related to the processing times, product flow, working loads, product distribution, sizes, etc, were available

- the operation ways were clearly established and posed no major modelling problems

- other data were obtained by observation and measurement, and were agreed with the customer.

As regards other operations, only those that are relevant for the sorting system performance were modelled. For example, due to the unavailability of historical data for the transport times and deliveries, etc, a uniform distribution was used to model, for instance, the entering flow to the sorter. As neither maintenance procedures nor equipment failures influence significantly the regular operation of the system, these were ignored.

Basically, the performing of the sorter is as follows. When an entering item is assigned a destination by the inspection stations, if all the lanes are free and that is the 
first PSL to that outlet, any lane is assigned. All the subsequent items to that location will be diverted to that outlet. If the spur is full, the item will circulate until there is room in it. When there are enough items to build a pallet at that lane, the next PSL to the same location will be diverted to a different lane, if there is any.

The entering flow of products in the conveyor system (25500 PSLs/day) and the load distribution (a table that relates the destination to the incoming load) reflect a forthcoming situation of the pharmaceutical company. Other parameters have been fixed based on the information given by the enterprise, such as:

- A shipping pallet consists of 29 PSLs and it requires an average of 5 minutes to be packed.

- The speed of the conveying system is $2 \mathrm{~m} / \mathrm{s}$.

- Operator speed $=1 \mathrm{~m} / \mathrm{s}$ (distance between lanes $=$ $0.5 \mathrm{~m})$

- The enterprise proposed to consider the next three shifts. The 8 hours night one with two operators; the main one of 7 hours, and a third one of 3.5 hours. The number of operators of these two shifts depends on the proposal.

- The length of a PSL is $1 / 3 \mathrm{~m}$ on the average.

- Different destinations of the new distribution centre: 150 (i.e., minimum number of lanes).

Initial conditions were considered.

The model was verified and validated using different techriques [12]. In fact, as a part of the model verification process, a simulation model was developed for a simpler sorter that is currently working in another logistic centre of the same enterprise. The results of the simulation were compared to the actual production data to validate the model and the collected measures.

On the other hand, the members of the company who accompanied the project were crucial in this process, as their knowledge of the sorting process details allowed for the detection of modelling errors, and also contributed to assure that the model was faithfully reproducing the operational procedures they wanted for the new distribution system, by examining model execution traces.

\section{A. Evaluation indexes}

The goal of this simulation study is to analyse the impact of some proposed alternatives on the overall performance of the system. The selected performance measures to evaluate the impact of these proposals are the following,

i) Number of working hours (WH): number of real hours that all the operators have been packing (Packing Time, PT) and attending the lanes (Time in Transit, TT). It is a measure of the true spent time.

ii) True Work (TW): the percentage of the executed work vs. the nominal one. The latest is calculated adding the number of operators times number of hours for each shift. For example, for the proposal of 12 operators for the second and third shifts,

$8 h \times 2$ op $\left(1^{\text {st }}\right.$ shift $)+7 h \times 12$ op $\left(2^{\text {nd }}\right.$ shift $)$

$$
+3.5 \text { hx } 12 \text { op }\left(3^{\text {rd }} \text { shift }\right)=142 \text { hours (nominal) }
$$$$
94.5 \% \text { (TW) of } 142 h=134.2 \mathrm{WH}
$$

iii) Circulation Peak (CP): the maximum number of items that were found at the main conveyor of the sorter at a specific moment. iv) Circulation Index (CI): the average of PSLs that have been on the sorter at any moment.

These performance parameters proved critical for the enterprise. Besides, some other measurements have been obtained during the experiments, including the time spent by the products on the conveyor, the time a lane has been blocked, if any, etc.

The random nature of simulation inputs renders the simulation runs to produce a statistical estimate of the performance measures not the measures themselves.

\section{B. Simulation Results}

Next, the simulation models were executed for an entire day (three shifts). The completely dynamic destination-lane, with partially dynamic operator-lane assignment has been implemented, with the one-to-one packing operation mode for the workforce.

For each run three parameters have been varied:

- Number and length of the lanes.

- Number of operators in the second and third shifts.

Some results of the simulation are presented here. Each of the parameters related in Section IV.A is analysed and the different layouts are compared.

The operators $(12,14,16,18$, or 20$)$ are evenly distributed on the sides of the sorter and they are supposed to stay on that side. The divert locations of the system are $160,170,180$, or 197 ; taking into account that the number of different destinations is 150 , extra lanes have been considered in all the cases.

The capacity (number of items) of each lane, i.e., its length, varies from $3 \mathrm{~m}$ to $10 \mathrm{~m}(6 \mathrm{~m} \approx 18 \mathrm{PSLs})$. It is worth noting that as an average of 29 PSLs are needed to complete a pallet, most of the spurs that have been simulated are not large enough to contain all of them simultaneously.

The values presented in the next figures correspond only to the intermediate shift, as it is the worst case because of the highest flow of products.

Fig. 3 shows the packing time (' $y$ ' axis) when different number of operators, lanes, and length are considered. As it can be expected, the packing time decreases when more operators are working. This parameter hardly varies according to the number of lanes and the lenght. The length of the lanes does affect when there are very few operators, but no significantly. On the whole, the time that operators spend packaging is almost independent on the number and length of the ramps and depends on the number of operators.

Regarding time in transit, if there are many operators each of them will be in charge of fewer outlets. Thus, they have to change the lane that they are attending to more often because of the one-to-one packing mode. As it is possible to prove, this parameter also depends on the number and length of the spurs. It is important to remark that when there are enough operators, the time in transit does not vary.

Taking into accout both parameters, PT and TT, it is possible to notice that if fewer operators, the operator percent utilisation value is higher. In fact, the performance is very poor for many operators. This parameter, the true work, does not vary apparently with the number and length of the lanes (Fig.4). 
Packing time (\%)
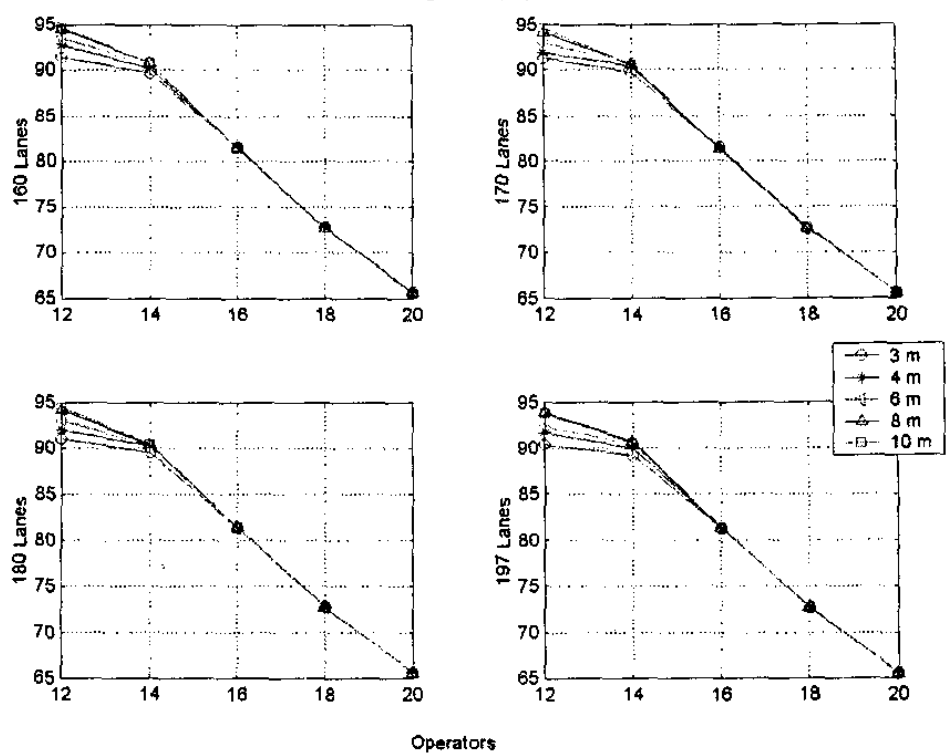

Fig. 3 Packing time (\%) vs. number of operators for different number of lanes and different lengths
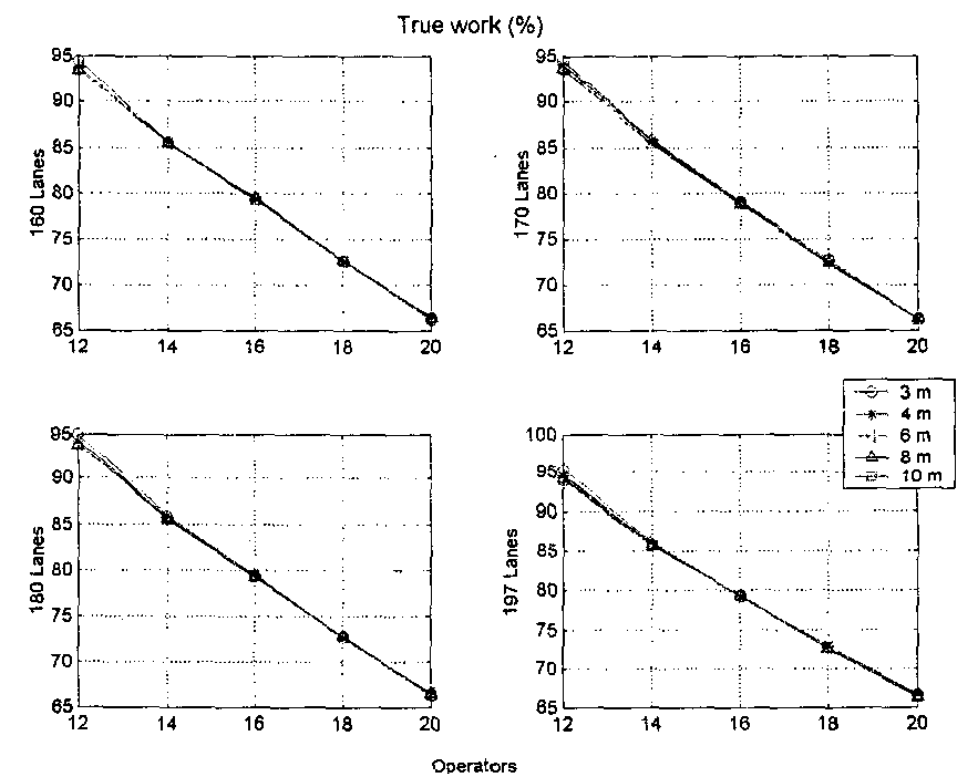

Fig. 4 True work (\%) vs. number of operators for different number of lanes and different lengths

Another interesting measurement refers to the circulation of items on the sorter because the enterprise was very interested in avoiding or reducing the circulation. The most significant one regards the circulation index (Fig. 5), although the curve for the circulation peak (CP) follows the same shape. The $\mathrm{Cl}$ depends mainly on the length of the lanes and the number of operators. It is also important to notice that the different number of lanes used in the proposals does not affect this parameter significantly.
The circulation average is significantly high only when there are very few operators. On the curve, it is possible to notice that there are two signifcant changes at 14 and 16 operators. The first one means the transition from a hardly feasible configuration to another that is viable. The second one represents a step forward in reaching an optimum solution. In fact, for more than 16 operators there is no circulation. So, if trying to avoid circulation, this can be 
acomplished by increasing the the workforce even though their performance will be poorer.
The same non-linear behaviour was shown in Fig. 3 for the packing time. In that case, the inflexion point occurs only when working with 14 operators.
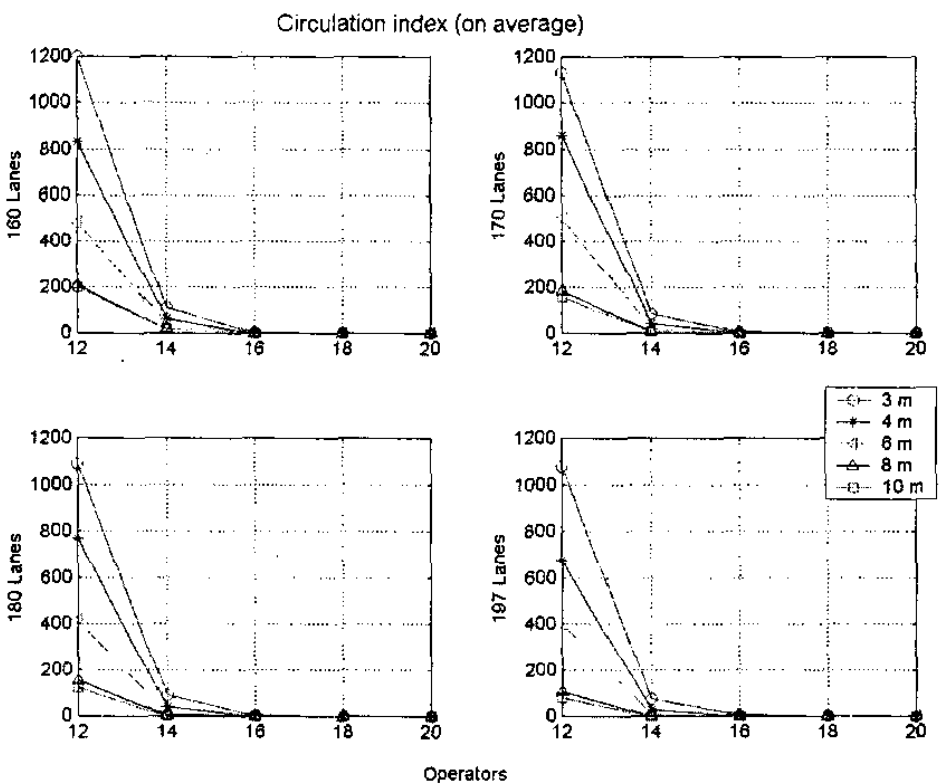

Fig. 5 Circulation Index (on average) vs. number of operators, for different number of tanes and different iengths

Focusing on these two proposals, Fig. 6 shows the results of the $\mathrm{Cl}$ of the model execution when changing the number and length of the lanes. When working with a group of 14 , the operator percent performance value is high although the probability of circulation is also higher. Therefore, this configuration requires larger lanes. If 16 operators are working, shorter spurs can be used and the circulation is still low, but the workforce performance is worse.

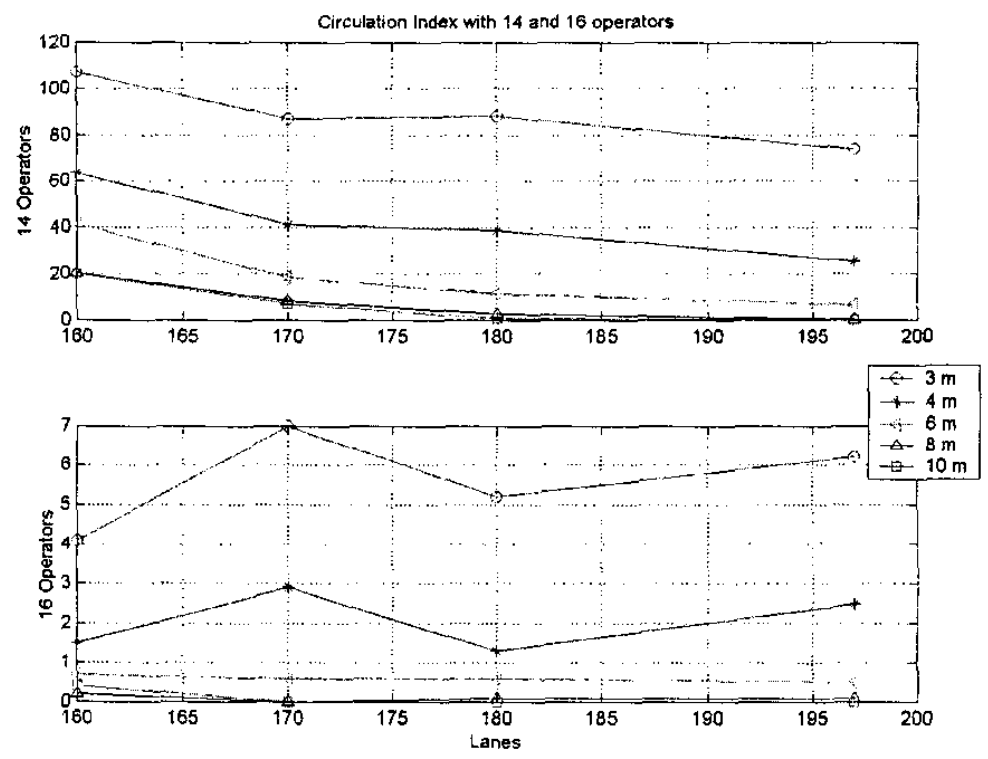

Fig. 6 Circulation Index with 14 and 16 operators vs. number of lanes for different lengths 
Taking into account all the results of the simulation runs, the general conclusions can be summarized as follows,

a. Length of the lanes: when shorter, the results are worse in the sense of more possible circulation and therefore, more operators needed to prevent items continuous circulating and to empty the lanes. However, if the entering flow is low or there are enough operators, decreasing the length does not affect so much the results, and from the economic point of view, the shorter spurs are cheaper. In fact, nowadays, the standard length is approximately $6 \mathrm{~m}$, and it is not common to find larger ones. The results show that using that length (capacity: 18 PSLs), the system performance is satisfactory when working with 16,18 or 20 operators and not too many spurs over the number of destinations $(160,170 \ldots)$.

b. Number of operators: it is concluded to be the most contributing factor in a well-dimensioned situation (enough lanes and appropriate length). If there are many operators, the circulation is reduced but the workforce utilisation also decreases. In general, for any number of outlets and whatever length, it is always possible to avoid circulation by increasing the number of operators.

c. Number of spurs: this has been shown as the less important factor, supposing the number of lanes is enough (that means, at least one for each outlet destination and some extra, as the company required). Its choice is strongly related to the number of outlets and the entering flow to the sorter. In general, items circulation is produced more by the length of the lanes than by the number. The number of extra lanes that are needed has been also studied for different situations.

However, there are other major conclusions, such as the relationship between the circulation and the qualitative parameter Available Zone ( $A Z$ ). When increasing $A Z$ by establishing a completely dynamic assignment, the circulation on the sorter decreases as the items are sorted more quickly, etc.

In order to take a decision, the most significant information comes out from the relationship between the number of operators, the number of lanes and their length (the cost of the solution), the percentage of operators' utilisation (percentage of performance), and the circulation (robustness of the configuration). An analytical function that relates these three parameters is difficult to achieve, but simulation has helped to get an insight into it.

After the simulation runs and the analysis of all the proposals, the main conclusion to reduce costs and to avoid circulation is that the best strategy for the sorter is the one described as "Completely dynamic Destination-Lane, with Partially dynamic Operator-Lane Assignment", and the layout of $6 \mathrm{~m}$ length lanes and 16 operators packing one-toone.

\section{CONCLUSIONS}

The main aim of this simulation was to assess a set of feasible proposals of a new distribution centre to the pharmaceutical company. The study is focused on the working procedures, in the attempt to increase the system throughput and overall productivity.

For that purpose, a simulation model of the current distribution process has been developed, and the relevant performance measures from the simulation outputs allowed us for the identification of a set of operational changes to the sorting procedures. The outcome of the study was then discussed with the management of the involved company and a set of realistic modifications to the sorter was agreed.

These proposals were included in the simulation models of the new distribution centre. The simulation models resulted in a more in-depth understanding of sorter parameters and its configuration.

Computational results and analysis are presented. Through these simulation experiments, it was found that the improvement achieved by choosing a specific sorting strategy can be significant. The study shows also the number of operators required and the specification of the working procedures to be used, showing some interesting non-linear behaviours.

On the other hand, the overall simulation confirmed a better understanding of some of the concerns addressed earlier for the company.

This study has helped to make a decision about the supplier, which has been chosen taking into account this analysis. The company's goals have been fully attained and, to best prove it, all the suggested proposals to the new distribution centre are being implemented.

\section{REFERENCES}

[1] A.M. Law and W.D. Kelton, Simulation, Modelling and Analysis, Mc Graw-Hill Int. Editions; 2000.

[2] M. Manivannan, "Simulation of Logistics and Transportation Systems", in Handbook of Simulation, 1998, pp. 227-229.

[3] F.P. Biemens and C.A. Vissers, "Reference Model for Manufacturing Planning and Control", Journal of Manufacturing Systems, 8(1), 1989, 35-46.

[4] D.J. Medeiros, "Flexible Control for Manufacturing Systems: a Simulation-Based Approach", in Progress in Material Handling Research, NC, 2000, pp. 217-224.

[5] S. Miller, "Introduction to Manufacturing Simulation", in Proceedings of the 2000 Winter Simulation Conference, 2000, pp. 63-66.

[6] A.M. Law and M.G. McComas, "Simulation of manufacturing systems", in Proceedings of the 1998 Winter Simulation Conference, 1998, pp. 49-52.

[7] Banks, J. Carson and B.L. Nelson, Discrete-events simulation, Prentice Hall; 1996.

[8] S. Narayanan, D.A. Bodner, U. Sreekanth, T. Govindaraj, LF. McGinnis and CM. Mitchell, "Research in Object-Oriented Manufacturing Simulations: an Assessment of the State of the Art", IIE Transactions, 30(9), 1998, pp. 795-810.

[9] Taylor ED (Enterprise Dynamics) FlexSim Software Products Inc., (April 2003): //www. flexsim.com (no longer supported).

[10] R. Hullinger, "Taylor Enterprise Dynamics", in Proc. of the 1999 Winter Simulation Conference, 1999, pp. 227.229.

[11] GT. Mackulak and PA. Savory, "Ascertaining Important Features for Industrial Simulation Environments", Simulation, 63 , Oct 1994, pp. 211-221.

[12] R.G. Sargent, "Validation and verification of simulation models", in Proc. of the 1999 Winter Simulation Conference, 1999 , pp. 39-48. 